Published in final edited form as:

Curr Drug Abuse Rev. 2017 ; 9(2): 106-112. doi:10.2174/1874473709666161229121527.

\title{
Proceeding of the $8^{\text {th }}$ Alcohol Hangover Research Group Meeting
}

\author{
Marlou Mackus ${ }^{1}$, Sally Adams ${ }^{2}$, Amir Barzilay $^{3}$, Sarah Benson ${ }^{4}$, Lauren Blau ${ }^{5}$, Jacqueline \\ Iversen ${ }^{6}$, Sean J. Johnson 7 , Ali Keshavarzian ${ }^{8}$, Andrew Scholey ${ }^{4}$, Gordon S. Smith ${ }^{9}$, \\ Constantine Trela ${ }^{10}$, Vatsalya Vatsalya ${ }^{5}$, and Joris C. Verster ${ }^{1,4,{ }^{*}}$
}

\begin{abstract}
${ }^{1}$ Utrecht University, Utrecht, The Netherlands ${ }^{2}$ University of Bath, Bath, UK ${ }^{3}$ Vital Beverages, Lod Northern Industrial Zone, Israel ${ }^{4}$ Swinburne University, Melbourne, Australia ${ }^{5}$ University of Louisville, Louisville, KY 40208, USA ${ }^{6}$ Sen-Jam Pharmaceutical, USA ${ }^{7}$ University of the West England, Bristol, UK ${ }^{8}$ Rush University Medical Center, Chicago, IL 60612, USA ${ }^{9}$ University of Maryland, Baltimore, MD 21201, USA ${ }^{10}$ University of Missouri, Missouri, Columbia MO 65211 , USA
\end{abstract}

\begin{abstract}
Alcohol hangover is one of the most commonly experienced consequences of alcohol consumption. An alcohol hangover develops as the blood alcohol concentrations (BAC) approaches zero, and is characterized by a general feeling of misery. More insight into the pathology of an alcohol hangover needs to be gained, in order to enhance the understanding of the area, and as a potential contribution to the innovation of a preventative or hangover curing treatment. The Alcohol Hangover Research Group (AHRG) was founded to support the area of alcohol hangover. This proceeding describes the latest findings in the area of alcohol hangovers, and future research plans, discussed at the $8^{\text {th }}$ Alcohol Hangover Research Group meeting, held on June 25, 2016, New Orleans, USA. Novel insight in potential causes, consequences, and treatment of alcohol hangover were revealed during the meeting, as well as further research plans to examine biomarkers of recent alcohol consumption, immune functioning, alcohol metabolism, and potential treatments.
\end{abstract}

\section{Keywords}

Alcohol; ethanol; hangover; treatment; cognitive impairment; potential therapeutics

\footnotetext{
*Address correspondence to this author at the Utrecht University, Utrecht Institute for Pharmaceutical Sciences, Division of Pharmacology, Universiteitsweg 99, 3584CG, Utrecht, The Netherlands; Tel: +31 30253 6909; Fax: +31 30253 7900; j.c.verster@uu.nl.

Conflict of Interest: Sean Johnson has undertaken sponsored research for Pfizer, AstraZeneca, Merck, Gilead, Novartis, Roche, and Red Bull GmbH. Andrew Scholey has held research grants from Abbott Nutrition, Bayer Healthcare, Cognis, Cyvex,

GlaxoSmithKline, Kemin, Naturex, Nestlé, Martek, Master-foods, and Wrigley, and has acted as a consultant/expert advisor to Abbott Nutrition, Barilla, Bayer Healthcare, Danone, Flordis, GlaxoSmithKline Healthcare, Masterfoods, Martek, Novartis, Unilever, and Wrigley. Sarah Benson received research support from Red Bull. Ali Keshavarzian received research support from grants R01AA023417 and R01AA020216. Joris Verster has received grants/research support from the Dutch Ministry of Infrastructure and the Environment, Janssen Research and Development, Nutricia, Takeda, and Red Bull and has acted as a consultant for the Canadian Beverage Association, Centraal Bureau Drogisterijbedrijven, Coleman Frost, Danone, Deenox, Eisai, Janssen, Jazz, Purdue, Red Bull, Sanofi-Aventis, Sen-Jam Pharmaceutical, Sepracor, Takeda, Transcept, Trimbos Institute, and Vital Beverages. The other authors have no conflicts of interest to declare.

Send Orders for Reprints to reprints@ benthamscience.ae
} 


\section{Introduction}

One of the most commonly experienced negative consequences of alcohol consumption is the alcohol hangover. According to definition, the alcohol hangover commences once the blood alcohol concentration (BAC) returns to zero and is characterized by a general feeling of misery lasting up to 24 hours.

The alcohol hangover has profound negative socioeconomic consequences [1, 2]. For example, in 2010 the US Centers for Disease Control and Prevention estimated a yearly loss of 179 billion US\$ related to reduced productivity due to alcohol consumption. Despite the large socioeconomic and health costs associated with alcohol hangovers, the area receives relatively little research interest and there is limited understanding of the pathology of an alcohol induced hangover. A deeper understanding of the pathological changes that occur during a hangover are needed to enhance our understanding of the area and potentially contribute to the development of a hangover prevention or cure.

In support of the area of alcohol hangover research, the Alcohol Hangover Research Group (AHRG) was founded in 2010 with the aim of uniting international researchers to collaborate on projects, improve the quality and methodology of hangover research, and share knowledge [1]. The current proceedings provide an overview of the recent research and future research plans that were discussed during the $8^{\text {th }}$ AHRG meeting, held on June 25, 2016, New Orleans, USA.

\section{Cognitive Effects of the Alcohol Hangover}

An alcohol hangover may result in impaired cognitive performance [3-5], negatively affecting the completion of everyday activities such as job performance [2] and driving [6]. Within laboratory settings, mixed effects have been found on simple tests of short duration that require little effort and/or cognitive demand. The use of various methodological designs across these studies may have contributed to these inconsistencies and make direct comparisons of findings and interpretations of results difficult [1,3]. More demanding tests that require cognitive resources for a sustained period, such as those measuring executive functioning, division of attention, and driving simulator performance, often show significant impairment.

Amir Barzilay (Vital Beverages, Israel) discussed several potential methodological shortcomings to be considered when designing alcohol hangover research. Of interest, Barzilay's experimental data revealed that alcohol elimination rate varies with the achieved peak breath alcohol concentration (BrAC), with quicker alcohol metabolism following higher BrACs compared to lower BrACs. Several other factors may also affect the rate of alcohol metabolism and include the consumption of fatty food which slow absorption rate and in accordance, reduce the achieved peak BrAC. Therefore, future laboratory studies should implement a strict 24-hour fat-free diet to ensure that significant confounding variables are reduced. 
Andrew Scholey (Swinburne University, Australia) presented preliminary data from an onpremise study whereby data was collected from patrons leaving a central entertainment district of an Australian state capital and again, the following morning while the patrons were experiencing a hangover state. During the evening, BrAC measurements were collected and in the morning, self-reported drinking behavior during the previous night was recorded and participants completed a short online cognitive task. Hangover severity was assessed using the Hangover Severity Scale (AHSS: [7]) and significantly correlated with previous night's $\mathrm{BrAC}(\mathrm{r}=0.228, \mathrm{p}=0.019)$. Speed of completing the Trail Making Test $\mathrm{B}$ task was significantly correlated with hangover severity $(\mathrm{r}=0.245, \mathrm{p}=0.012)$, previous night's $\mathrm{BrAC}$ $(\mathrm{r}=0.0197, \mathrm{p}=0.041)$, and time spent drinking $(\mathrm{r}=0.376, \mathrm{p}<0.001)$ while accuracy was not affected. These findings are the reverse to the typical pattern of results seen during intoxication whereby reaction times remain unchanged but error rates increase, characteristic of a speed-accuracy trade-off.

On-premise designs are becoming increasingly popular within the hangover field. Alternative methodologies used in the hangover field are generally either laboratory studies or surveys completed during a hangover state. While both methodologies can be advantageous depending on the research aims, on-premise designs provide strong ecological validity and are able to capture real night-life behavior. In laboratory settings, alcohol consumption is generally capped at levels lower than consumed on a night out and variables affecting hangover, such as sleep and type of alcohol consumed, are often controlled. Survey methodologies are limited by relying on participant recall, particularly in estimating $\mathrm{BrAC}$ where participants who consumed large amounts of alcohol would most likely not remember all of their drinks.

Cognitive impairment caused by alcohol induced hangovers can have serious consequences on daily activities. A driving simulator study has shown that highway driving is significantly impaired during the hangover state [8] and yet, $56.4 \%$ of the interviewed Dutch truck drivers admitted driving, both privately and professionally, while in a hangover state [6]. However, as most data was gathered using self-report surveys and laboratory experiments, little is known about real life consequences, including the actual risk of injury.

Gordon Smith (University of Maryland, USA) discussed his ongoing research assessing the risk of injury while driving during a hangover state. As part of a larger study by Smith and colleagues, the prevalence of hangovers among seriously injured sober drivers was estimated. To identify and quantify the role of hangovers in motor vehicle crashes (MVC) ethyl glucuronide $(\mathrm{EtG})$ and ethyl sulfate $(\mathrm{EtS})$ bio-marker evidence of recent alcohol consumption was used to identify possible hangovers among sober (BAC zero) MVC drivers. Urine samples were collected from injured drivers admitted to the EMS Shock Trauma Center (University of Maryland) who gave consent and from all drivers killed in MVCs across the state for whom frozen urine samples were available from the Maryland Office of the Chief Medical Examiner (OCME). In those patients who consented, their leftover blood from routine testing was analyzed for the presence of illicit drugs. A total of 201 drivers admitted to the trauma center have been tested so far and approximately $22 \%$ of all drivers who had a BAC of zero when they were injured in a crash had biomarker evidence of recent drinking. Evidence of recent heavy drinking by EtG when BAC is zero is also greater 
in fatalities, especially in very heavy recent drinking. However, Smith and colleagues found that none of the drivers that used drugs had levels of EtG that indicated they were likely to have hangovers. Thus, drug use and alcohol hangovers do not seem to be related, although more cases are being tested and the results are thus only preliminary at this time.

\section{Determinants of the Alcohol Hangover}

The presence of an alcohol hangover and its corresponding symptoms greatly differ between individuals and it is unclear which factors determine the development and severity of a hangover. Differences in gender, age and ethnicity have been suggested as determinants for hangover severity [9]. It has also been suggested that a low sensitivity to alcohol may result in hangover immunity and thus, may contribute to continued heavy alcohol consumption [10]. Previous research has shown that a lower sensitivity to alcohol is associated with a significant increase in the risk of future alcoholism [11] and may predict alcohol-related blackouts [12].

Lauren Blau (National Institutes on Alcohol Abuse and Alcoholism, USA) discussed predictors of alcohol hangover, including drinking history and alcohol sensitivity in nondependent drinkers. Blau and colleagues used a Computer Assisted Infusion System (CAIS) where participants could self-administer alcohol intravenously (IV) and BrACs were controlled using a physiologically-based pharmacokinetic model-based algorithm [13]. Each session consisted of a 10-minute priming phase in which participants were instructed to press a button allowing four titrated amounts of IV alcohol to achieve a target BrAC of $0.03 \%$. Following a 15 -min rest, participants underwent a 2-hour "open bar" phase in which they were instructed to recreate a typical drinking experience. Participants were allowed to administer alcohol up to a $\mathrm{BrAC}$ of $0.1 \%$; and subjective responses to alcohol were collected at 15-minute intervals throughout the study. These included a modified Drug Effects Questionnaire (DEQ: [14]), which assessed response to alcohol on 5 items: 'like', 'want', 'feel', 'high', and 'intoxicated'. Hangover symptoms were assessed for the period between participants' departure from the study unit and 10AM the next morning, using the Alcohol Hangover Scale [15], along with questions related to craving and alcohol consumption following the study session. A total of $78 \%$ of participants endorsed having at least one hangover symptom following IV-ASA. The most commonly reported items were tiredness (67\%), thirst (57\%), and headache (32\%), and hangover (25\%). There was a wide variation in peak BrACs reached during the IV-ASA session; however, there was no correlation between peak BrAC and hangover scores. Hangover scores positively correlated with the dependence and harmful involvement subscales of the AUDIT and with the DEQ items 'feel' ( $\mathrm{p}=.02)$, 'high' $(\mathrm{p}=.04)$, and 'intoxicated' ( $\mathrm{p}=.004)$. Thus, risky drinking patterns were positively associated with hangover symptoms in non-dependent drinkers and this relationship was independent of the amount of alcohol consumed. Sensitivity to alcohol, however, was positively correlated with the experience of hangover symptoms. In other words, higher sensitivity to alcohol was related to experiencing more severe hangover symptoms.

Sally Adams (University of Bath) discussed qualitative data from a study examining student experiences of hangover. Findings indicated that hangover was associated with the 
experience of psychological symptoms, including cognitive impairment and mood effects. Responses from a public panel of social drinkers also revealed reported effects of hangover on performance of everyday activities, and in particular increased feelings of anxiety. Informed by these findings, Adams and colleagues are planning a series of experiments assessing biomarker and subjective measures of hangover to determine the effects of alcohol hangover on physical, subjective, and cognitive measures of anxiety, risk-taking, decision making and impulse control.

Constantine Trela (University of Missouri, USA) presented preliminary work examining the frequency of hangover following naturalistic drinking episodes in a sample of outpatients with Borderline Personality Disorder (BPD) and community controls (COM). Rationale for examining this group of patients was that substance abuse is a common co-morbid condition of BPD. Trela and colleagues demonstrated that BPD and COM drinkers did not differ in peak estimated BAC (using Mathews \& Miller's 1978 calculation) during these episodes, but that BPD drinkers demonstrated a steeper increase in estimated BAC relative to the start of their drinking episode. BPD drinkers were more likely to report a hangover following drinking relative to COM drinkers. When rate of drinking was included as a mediator, the relationship between BPD status and hangover frequency was no longer significant. Additional exploratory analyses that included an index of past-year frequency of hangover symptoms [16], age and sex as covariates demonstrated a similar pattern. Rate of drinking remained a significant mediator of the relationship between BPD status and hangover frequency with the inclusion of these covariates, though the relationship was only partially mediated in the exploratory analyses. These initial analyses suggest how the BPD individuals' drinking pattern (i.e., drinking faster) may lead to an increased frequency of hangover. In turn, experiencing hangovers more frequently may help to elucidate the comorbidity of BPD and Alcohol Use Disorder.

\section{The Pathology of Alcohol Hangover}

As alcohol is treated by the human body as a toxin, a role for the immune system in hangover pathology has been suggested [17]. A study by Kim et al. (2003) showed that blood concentrations of several cytokine (i.e., interleukin (IL)-10, IL-12, and interferon (IFN)- $\gamma$ ) were significantly increased 13 hours after alcohol consumption, demonstrating an inflammatory response during the next day hangover state [18].

Marlou Mackus (Utrecht University, The Netherlands) determined concentrations of a range of pro- and anti-inflammatory cytokines in saliva collected during hangover and on a control day (no alcohol consumed). Saliva samples were collected from every participant hourly between 9 am and $4 \mathrm{pm}$. Saliva samples were analyzed for concentrations of proinflammatory cytokines IL- $1 \beta$, IL-2, IL-6, IL-8, GMCSF, IFN- $\gamma$, and TNF- $\alpha$, and antiinflammatory cytokines IL-4, IL-5, and IL-10. At 6 hours after the last alcoholic drink was consumed, a peak in pro-inflammatory cytokines could be identified that returned to control levels about 2 to 3 hours thereafter. In contrast, for anti-inflammatory cytokine concentrations, no elevation in concentration was seen, and no significant differences were observed between the hangover and control day. The results suggest that the immune response to alcohol is most evident in the first 9 to 10 hours after alcohol consumption. This 
should however be confirmed by future studies measuring cytokine levels in the first hours after alcohol consumption. As participants were asleep after the drinking session, this was not possible in the current study design. This should preferably be a daytime study, as in more naturalistic designs of hangover trials participants are asleep the first hours after stopping drinking, making assay collection more difficult. Taken together, the data support previous findings that the immune system is involved in the development of the alcohol hangover.

Ali Keshavarzian (Rush University, USA) hypothesized that changes in oral and intestinal microbiota composition could also play an important role in the pathology of alcohol hangovers. Several studies have shown that chronic alcohol consumption results in changes in microbiota composition in humans [19, 20] and rodents [21]. Dysbiosis, i.e. the alcoholinduced changes in microbiota composition, is characterized by an increased abundance of pro-inflammatory bacteria and a decrease in anti-inflammatory bacteria. In addition, a decrease in gut health promoting bacterial products like short chain fatty acids promoting intestinal barrier integrity has been demonstrated after chronic alcohol use. Indeed, it is not surprising that disruption of intestinal barrier integrity, a so called 'leaky gut', has also been reported in alcoholics and alcohol-fed rodents [22, 23]. The combined dysbiosis and gut leakiness result in endotoxemia and increased levels of systemic pro-inflammatory cytokines like TNF and IL-6 [19, 21, 23]. Thus, it is conceivable that the source of inflammatory state in alcohol hangover and increased level of salivary IL- 6 is caused and/or moderated by changes in microbiota.

Of note, it is interesting that not all subjects complain of hangover after an alcohol binge. Indeed, it is now well known that not all alcoholics develop dysbiosis or gut leakiness [19, $20,22]$. It was recently showed that disruption of circadian rhythms is the risk factor for developing dysbiosis and gut leakiness in both alcohol-fed rodents and human alcoholics. It is intriguing that circadian rhythms appears to be important in developing hangover and that a synchronized circadian clock is critical for hangover recovery in rodents [24].

Studies have shown that a propensity to alcoholism and several other mental disorders are linked to dopamine D2 and serotonin pathways [25]. A therapeutic agent that could reduce the reinforcement of drinking [26] could effectively reduce alcohol intake. One of such postulated mechanisms for treatment is regulation of gut bacterial dysbiosis. Gut dysbiosis results in increased intestinal permeability leading to symptoms of hangover, supporting a role of the gut-brain axis as mediated by the microbiome [27]. Pathways underlying the gutbrain axis are multiple and highly complex, involving brain biochemistry and neuroinflammatory agents. Activation of sCD14 associated inflammation [18], and neuroinflammation [28] and neurotoxicity [29], are known confounding factors in other mental disorders, though not tested in alcohol use disorder. Nonetheless, one study has shown a close association between behavioral markers of alcohol dependence severity and markers of neuro-inflammation [30]. Larger studies might be able to investigate the mechanisms of endotoxemia [31] and pro-inflammatory responses that could be contributing to the adaptive reinforcement properties in alcohol addiction, which could be instrumental in the reinforcing properties of heavy alcohol drinking. 


\section{Potential Therapeutics}

Probiotics are known to reduce gut permeability and inflammation, and have been studied in liver disease. Probiotics could therefore be a useful treatment for lowering of alcohol intake by attenuating the endotoxemia-dependent elevation of neuro-inflammatory pathways [32].

Vatsalya Vatsalya (University of Louisville, USA) investigated differences in amount and pattern of drinking and corresponding changes in hangover symptomology between moderate and very heavy drinkers. Six male and female very heavy drinking patients within the age group of $21-70$ years old were enrolled in this probiotic treatment pilot study. Very heavy drinking was defined as consumption of 15 drinks per drinking day in these patients. A sample of 24 young (21-30 yrs.) male and female moderate drinkers who drank two to three drinks per drinking day were also included for comparative analysis of hangover symptomatology. Data for demographics, drinking history measures (Timeline Follow Back, Lifetime Drinking History, alcohol use questionnaire (for monthly evaluation), hangover scale, and family genogram) were recorded. Drinking history during the treatment was also collected. Baseline levels of the drinking measures were examined along with hangover scores between the moderate and very heavy drinkers. Three-month and six-month evaluation of alcohol consumption and responses for hangover were assessed as endpoints for treatment efficacy.

A substantial lowering of alcohol intake from 15 drinks to six drinks was observed after three months of treatment in individuals who also exhibited reduced inflammation as characterized by lowering of cytokines, and injury in the liver. Consequently, after three months, such patients also did not report any hangover symptoms after a drinking episode. No further improvements were seen at the six-month evaluation.

Amir Barzilay (Vital Beverages, Israel) discussed the results of a series of experiments to test the efficacy of a newly developed rapid action recovery product from a variety of symptoms following alcohol consumption to peak BAC levels between $0.100 \%-0.140 \%$. Based upon the scientific literature, a new combination treatment was developed consisting of several natural active ingredients that have a positive and rapid (15-30 minutes post consumption) effect on various hangover related symptoms such as motor functions, concentration, headache, nausea, stomach ache and tiredness. In addition, the product is aimed at speeding up natural alcohol metabolism rates in support of producing rapid recovery from a high BAC. The rationale of this product, to be taken shortly after stopping alcohol consumption, is three-fold: (1) quick recovery of rational behavior, cognitive and motor function (2) preventing short-term and the day after hangover symptoms; and (3) faster elimination of alcohol from the body. Comparing the product to placebo and control (alcohol without intervention), the data suggest that the product was capable of significantly enhancing the natural rate of alcohol metabolism. The severity of hangover symptoms was significantly reduced after taking the product. Finally, alcohol-impaired cognitive and motor functions, as measured by a battery of computerized cognitive tests, were significantly improved almost to Baseline (e.g. prior to alcohol consumption) levels after taking the product in comparison with Placebo and Control. 
Jacqueline Iversen (Sen-Jam Pharmaceutical, USA) discussed initial clinical data from another new hangover product, JMI-001, which should be taken before a drinking session. The goal of the product, which is a combination of an NSAID and an H1-receptor antagonist, is to prevent the presence or reduce the severity of core hangover symptoms such as headache, thirst, and fatigue. Data from a first small clinical trial revealed that, compared to placebo, the product was capable of reducing overall hangover severity as measured by the AHS total score, as well as individual symptom scores of headache, dizziness, and nausea. Although positive effects were evident, possibly due to the small sample size of this pilot study $(\mathrm{N}=13)$, the differences between placebo and JMI-001 were not statistically significant. Future studies are planned to further investigate the safety and efficacy of JMI-001 in the prevention of alcohol hangover.

\section{Hangover Immunity}

Previous experimental and survey data, suggests that up to $23 \%$ of the population may be resistant to the effects of alcohol hangover [33]. However, little is known about the phenomenon of hangover immunity. A closer look at the drinking behavior of Dutch students who claim never to have hangovers revealed that a large number of them simply do not consume enough alcohol to develop a hangover in the first place [34]. That is, the data revealed that $79 \%$ of them had an estimated peak BAC below $0.10 \%$ on their heaviest drinking occasion.

Sean Johnson (University of the West England, Bristol, UK) further explored the extent to which hangover immunity was reported among UK students. Of the 1321 students that completed the survey, $15.7 \%$ claimed to have not suffered a hangover (hangover negative) in the past year. The higher the students' estimated BAC, the less likely it was that they claimed to be hangover negative. Nearly half $(46.6 \%)$ of students who had not experienced a hangover in the past year had an estimated BAC below $0.08 \%$, with the percentage of those claiming to be hangover negative decreasing rapidly with higher estimated BACs. A cumulative percentage of hangover negative students revealed that $65.9 \%$ had an estimated BAC at or below the UK drink driving limit $(0.08 \%)$ and $80.3 \%$ were at or below the commonly held estimated BAC needed to develop a hangover $(0.11 \%)$ [1]. These findings provide further support that those who claim hangover immunity are simply not consuming enough alcohol to produce next-day hangover effects.

Joris Verster (Utrecht University, The Netherlands) compared 18 hungover drinkers with 18 hangover-immune drinkers in a naturalistic study, following them over the course of a drinking day and an alcohol-free evening. The most common and severe symptoms in the hangover group were sleepiness, tiredness, thirst, headache, concentration problems, nausea, clumsiness, dizziness, and stomach pain. In contrast, the hangover-immune group reported only modest increases in sleepiness, being tired, concentration problems, and thirst, without any relevant effects on more disabling symptoms such as headache, nausea, vomiting, and dizziness [35]. In both groups, urine samples were collected at 09.30 in the morning. These were analyzed for the presence of ethanol. In the hangover group, urine ethanol concentrations were significantly higher compared to the hangover-immune group. Urine ethanol concentration correlated significantly with overall hangover severity and that of 
various individual symptoms, including nausea, headache, and concentration problems [36]. The differences in urine ethanol concentration between the hangover group and the hangover-immune group suggest a relationship between ethanol metabolism and the risk of developing alcohol hangovers. Therefore, future research into hangovers amongst slow and fast metabolizers of alcohol is warranted.

\section{Discussion}

The data presented at the $8^{\text {th }}$ Alcohol Hangover Research Group meeting revealed new insights in the causes, consequences, prevention, and treatment of alcohol hangover. As data on hangover pathology has grown over recent years, efforts to develop an effective hangover cure is becoming increasingly supported by scientific support. Whereas in the past antihangover products were generally not accompanied by a scientifically credible rationale of efficacy, the presentations at this meeting showed different approaches to combat the alcohol hangover each with a clear and biologically plausible rationale.

Recent research has confirmed an important function of the immune system in hangover pathology [37] and differences in alcohol metabolism between those who experience hangovers and those who claim hangover-immunity [36]. As a result of an alcohol-induced dysbiosis, alcohol can induce gut leakiness, causing the release of systemic proinflammatory cytokines. This way, hangover may be initiated via the gut-brain axis. However, alcohol also directly affects brain functions, and increased cytokine concentrations during hangover have been measured in blood [18], saliva, and urine [37]. One presentation at the hangover meeting focused on counteracting the immune response by means of combining and NSAID and $\mathrm{H}_{1}$-receptor antagonist, whereas another examined the effectiveness of probiotics to counteract the hangover state. Alternatively, a product was presented that focuses on speeding up alcohol metabolism and the reduction of peak BAC after alcohol consumption. Differences in urine ethanol concentrations between those who experience a hangover and hangover free drinkers [36], despite consuming the same large amount of alcohol, support this approach. More research assessing the pathology of alcohol hangover is needed to determine which approach or combination of approaches is most effective.

Comparing those who experience a hangover and hangover-immune drinkers may also provide insights into the pathology of the alcohol hangover. Research should examine alternative biomarkers of recent alcohol consumption, immune functioning, and alcohol metabolites. The knowledge obtained from these studies will likely enhance the development of effective hangover cures.

Several advances related to the causes, consequences, prevention, and treatment of alcohol hangover were discussed the $8^{\text {th }}$ Alcohol Hangover Research Group meeting and future research should further elucidate the pathology of the alcohol hangover.

\section{Acknowledgments}

The $8^{\text {th }}$ Alcohol Hangover Research Group meeting was held as satellite meeting of the $39^{\text {th }}$ conference of the

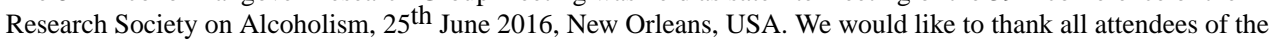


meeting who contributed to fruitful discussions, and the co-authors who contributed to the research presented at the meeting:

Chris Alford (University of the West England, Bristol, UK), Corey Allen (Centre for Human Psychopharmacology, Melbourne, Australia), Sarah Benson (Centre for Human Psychopharmacology, Melbourne, Australia), Faraz Bishehsari (Rush University Medical Centre, Chicago, USA), Karel Brookhuis (Groningen University, Groningen, The Netherlands), Ryan W. Carpenter (University of Missouri, Missouri USA), Grant Devilly (Centre for Human Psychopharmacology, Melbourne, Australia), Christopher Forsyth (Rush University Medical Centre, Chicago, USA), Johan Garssen (Utrecht University, Utrecht, The Netherlands), Jordy Kaufman (Centre for Psychopharmacology, Melbourne, Australia), Karen Knipping (Nutricia Danone, Utrecht, The Netherlands), Gerdien Korte-Bouws (Utrecht University, Utrecht, The Netherlands), Sean P. Lane (University of Missouri, Missouri USA; Purdue University, Indiana USUA), Aurora van de Loo (Utrecht University, Utrecht, The Netherlands), Craig J McClain (University of Louisville, Louisville, USA), Reinier van Neer (Utrecht University, Utrecht, The Netherlands), Thomas Nguyen (Utrecht University, Utrecht, The Netherlands), Thomas M. Piasecki (University of Missouri, Missouri USA), Vijay A Ramchandani (University of Louisville, Louisville, USA), Bethany Stangl (Section on Human Psychopharmacology, Louisville, USA), Garth Swanson (Rush University Medical Centre, Chicago, USA), Celeste Tipple (Centre for Human Psycho-pharmacology, Melbourne, Australia), Timothy J. Trull (University of Missouri, Missouri USA), X Wang (Utrecht University, Utrecht, The Netherlands), and Robin Voigt (Rush University Medical Centre, Chicago, USA).

\section{References}

1. Verster JC, Stephens R. The importance of raising the profile of alcohol hangover research. Curr Drug Abuse Rev. 2010:64-7. [PubMed: 20822482]

2. Frone MR, Verster JC. Alcohol hangover and the workplace: A need for research. Curr Drug Abuse Rev. 2013; 6:177-9. [PubMed: 24397467]

3. Stephens R, Grange JA, Jones K, Owen L. A critical analysis of alcohol hangover research methodology for surveys or studies of effects on cognition. Psychopharmacology. 2014; 231:222336. [PubMed: 24633471]

4. Ling J, Stephens R, M Heffernan T. Cognitive and psychomotor performance during alcohol hangover. Curr Drug Abuse Rev. 2010; 3:80-7. [PubMed: 20712592]

5. Stephens R, Ling J, Heffernan TM, Heather N, Jones K. A review of the literature on the cognitive effects of alcohol hangover. Alcohol and Alcoholism. 2008; 43:163-70. [PubMed: 18238851]

6. Verster JC, Bervoets AC, De Klerk S, Vreman RA, Olivier B, Roth T, Brookhuis KA. Effects of alcohol hangover on simulated highway driving performance. Psychopharmacology. 2014; 231(15): 2999-3008. [PubMed: 24563184]

7. Penning R, McKinney A, Bus LD, et al. Measurement of alcohol hangover severity: Development of the Alcohol Hangover Severity Scale (AHSS). Psychopharmacology. 2013; 225:803-810. [PubMed: 23007602]

8. Verster JC, Van der Maarel M, McKinney A, Olivier B, de Haan L. Driving during alcohol hangover among Dutch professional truck drivers. Traffic Inj Prev. 2014; 15(5):434-8. [PubMed: 24678564]

9. Penning R, Veldstra J, Daamen AP, Olivier B, Verster JC. Drugs of abuse, driving and traffic safety. Curr Drug Abuse Rev. 2010; 3:23-32. [PubMed: 20088818]

10. Rohsenow DJ, Howland J, Winter M, Bliss CA, Littlefield CA, Heeren TC, Calise TV. Hangover sensitivity after controlled alcohol administration as predictor of post-college drinking. J Abnorm Psychol. 2012; 121:270-5. [PubMed: 21859168]

11. Schuckit MA. Low level of response to alcohol as a predictor of future alcoholism. Am J Psychiatry. 1994; 151(2):184-9. [PubMed: 8296886]

12. Wetherill RR, Fromme K. Subjective responses to alcohol prime event-specific alcohol consumption and predict blackouts and hangover. J Stud Alcohol Drugs. 2009; 70:593-600. [PubMed: 19515300]

13. Zimmermann US, O'Connor S, Ramchandani VA. Modeling alcohol self-administration in the human laboratory. Curr Top Behav Neurosci. 2013; 13:315-53. [PubMed: 21792747]

14. Gilman JM, Ramchandani VA, Davis MB, Bjork JM, Hommer DW. Why we like to drink: A functional magnetic resonance study of the rewarding and anxiolytic effects of alcohol. Neurology of Disease. 2008; 28(18):4583-91. 
15. Rohsenow DJ, Howland J, Minsky SJ, Almeida A, Roehrs TA. The acute hangover scale: A new measure of immediate hangover symptoms. Addictive behaviors. 2007; 32(6):1314-20. [PubMed: 17097819]

16. Slutske WS, Piasecki TM, Hunt-Carter EE. Development and initial validation of the hangover symptoms scale: Prevalence and correlates of hangover symptoms in college students. Alcohol Clin Exp Res. 2003; 27(9)

17. Verster JC. The alcohol hangover-a puzzling phenomenon. Alcohol and alcoholism. 2008; 43(2): 124-6. [PubMed: 18182417]

18. Kim DJ, Kim W, Yoon SJ, Choi BM, Kim JS, Go HJ, Kim YK, Jeong J. Effects of alcohol hangover on cytokine production in healthy subjects. Alcohol. 2003; 31(3):167-70. [PubMed: 14693266]

19. Mutlu EA, Gillevet PM, Rangwala H, Sikaroodi M, Engen PA, Kwasny M, Lau CK, Keshavarzian A. Colonic microbiome is altered in alcoholism. Am J Physiol Gastrointest Liver Physiol. 2012; 302:G966-78. [PubMed: 22241860]

20. Engen PA, Green SJ, Voigt RM, Forsyth CB, Keshavarzian A. The Gastrointestinal Microbiome: Alcohol Effects on the Composition of Intestinal Microbiota. Alcohol Res. 2015; 37:223-36. [PubMed: 26695747]

21. Mutlu E, Keshavarzian A, Engen PA, Forsyth CB, Sikaroodi M, Gillevet P. Intestinal dysbiosis: A possible mechanism of alcohol-induced endotoxemia and alcoholic steatohepatitis in rats. Alcohol Clin Exp Res. 2009; 33:1836-46. [PubMed: 19645728]

22. Keshavarzian A, Holmes EW, Patel M, Iber F, Fields JZ, Pethkar S. Leaky gut in alcoholic cirrhosis: A possible mechanism for alcohol-induced liver damage. Am J Gastroenterol. 1999; 94:200-7. [PubMed: 9934756]

23. Keshavarzian A, Farhadi A, Forsyth CB, Rangan J, Jakate S, Shaikh M, Banan A. Fields JZ/ Evidence that chronic alcohol exposure promotes intestinal oxidative stress, intestinal hyperpermeability and endotoxemia prior to development of alcoholic steatohepatitis in rats. J Hepatol. 2009; 50:538-47. [PubMed: 19155080]

24. Karadayian AG, Lores-Arnaiz S, Cutrera RA. The effect of constant darkness and circadian resynchronization on the recovery of alcohol hangover. Behav Brain Res. 2014; 268:94-103. [PubMed: 24717330]

25. O'connor JC, Lawson MA, Andre C, Moreau M, Lestage J, Castanon N, Kelley KW, Dantzer R. Lipopolysaccharide-induced depressive-like behavior is mediated by indoleamine 2, 3dioxygenase activation in mice. Mol Psychiat. 2009; 14(5):511-22.

26. Koob GF, Roberts AJ, Schulteis G, Parsons LH, Heyser CJ, Hyytiä P, Merlo-Pich E, Weiss F. Neurocircuitry targets in ethanol reward and dependence. Alcohol Clin Exp Res. 1998; 22(1):3-9. [PubMed: 9514280]

27. Leclercq S, Matamoros S, Cani PD, Neyrinck AM, Jamar F, Stärkel P, Windey K, Tremaroli V, Bäckhed F, Verbeke K, De Ti-mary P. Intestinal permeability, gut-bacterial dysbiosis, and behavioral markers of alcohol-dependence severity. Proc Natl Acad Sci USA. 2014; 111(42):E4485-93. [PubMed: 25288760]

28. Walter S, Letiembre M, Liu Y, et al. Role of the toll-like receptor 4 in neuroinflammation in Alzheimer's disease. Cell Physiol Biochem. 2007; 20(6):947-56. [PubMed: 17982277]

29. Fassbender K, Walter S, Kühl S, et al. The LPS receptor (CD14) links innate immunity with Alzheimer's disease. FASEB J. 2004; 18(1):203-5. [PubMed: 14597556]

30. Umhau JC, Schwandt M, Solomon MG, et al. Cerebrospinal fluid monocyte chemoattractant protein-1 in alcoholics: support for a neuroinflammatory model of chronic alcoholism. Alcohol Clin Exp Res. 2014; 38(5):1301-6. [PubMed: 24689518]

31. Bode C, Kugler V, Bode JC. Endotoxemia in patients with alcoholic and non-alcoholic cirrhosis and in subjects with no evidence of chronic liver disease following acute alcohol excess. J Hepatol. 1987; 4(1):8-14. [PubMed: 3571935]

32. Parlesak A, Schäfer C, Schütz T, Bode JC, Bode C. Increased intestinal permeability to macromolecules and endotoxemia in patients with chronic alcohol abuse in different stages of alcohol-induced liver disease. J Hepatol. 2000; 32(5):742-7. [PubMed: 10845660] 
33. Howland J, Rohsenow DJ, Edwards EM. Are some drinkers resistant to hangover? A literature review Curr Drug Abuse Rev. 2008; 1(1):42-6. [PubMed: 19630704]

34. Verster JC, De Klerk S, Bervoets AC, Kruisselbrink LD. Can hangover immunity really be claimed. Curr Drug Abuse Rev. 2013; 6:253-4. [PubMed: 25311093]

35. Hogewoning A, Van De Loo A, Mackus M, et al. Characteristics of social drinkers with and without a hangover after heavy alcohol consumption. Subst Abuse Rehabil. 2016; 7:161-7. [PubMed: 27895524]

36. Van De Loo A, Mackus M, Korte-Bouws GAH, Brookhuis KA, Garssen J, Verster JC. Urine ethanol concentration and alcohol hangover severity. Psychopharmacology. 2017; 234:73-77. [PubMed: 27678552]

37. Van De Loo A, Hogewoning A, Raasveld SJ, et al. Immunological and mood changes the day after heavy alcohol consumption: A comparison of drinkers with a hangover versus those who claim hangover resistance. Drug Alcohol Rev. 2015; 34(Suppl 1):63. 